\title{
TRABAJO GRUPAL VS RESPONSABILIDAD INDIVIDUAL: REPARTO DE NOTAS
}

Working in groups VS individual responsibility: sharing grades

O trabalho em grupo vs responsabilidade individual: compartilhamento de notas

Jorge Garrote García (1)

Oscar Casado Berrocal (2)

David Hortigüela Alcalá (3)

Israel Herrán Álvarez (4)

Ángel Pérez-Pueyo (5)

(1) IES Lancia (León), España. Teléfono- +34 987259800. Correo electrónico: jorge.garrote@hotmail.com

(2) CEIP Ponce de León. oscar.casado.berrocal@gmail.com

(3) Universidad de Burgos. dhortiguela@ubu.es

(4) IES Doctor Sancho de Matienzo. israeleducacionfisica@gmail.com

(5) Universidad de León. angel.perez.pueyo@unileon.es

\begin{abstract}
Resumen
Esta propuesta se centra en el funcionamiento del trabajo grupal en una producción motriz de Educación Física, proponiendo diversas estrategias que permitan potenciar los aspectos positivos del mismo y minimizar los problemas más comunes. En primer lugar, se entrega y explica el instrumento de evaluación y calificación que se utilizará con intención formativa. A continuación, se establece una distribución de roles dentro del grupo para fomentar la responsabilidad individual. Posteriormente, se valorará la producción motriz a través de autoevaluación, coevaluación intergrupal y heteroevaluación. Finalmente, se recurrirá a un reparto de notas que permita que a través de una coevaluación intragrupal todos los alumnos obtengan la nota que merecen. Palabras clave: Reparto de notas; trabajo grupal; evaluación objetiva; instrumento de evaluación
\end{abstract}




\begin{abstract}
This proposal is focused on how to work in groups to create a motor production in Physical Education. Our aim is to propose different strategies that let us foster the positive aspects of group work while reducing the common problems associated with it. First, we hand in and explain the assessment and grading instrument so to provide a formative assessment. Second, we distribute roles among the group members to increase the individual responsibility. Later, the motor production will be assessed through selfassessment, peer-assessment among the groups and heteroassessment. Finally, there will be a final sharing of the grades through peer-assessment among the members of the group to be able to give all students the grades they deserve.
\end{abstract}

Keywords: Sharing grades; group work; objective assessment; assessment instrument

\title{
Resumo
}

Esta proposta centra-se sobre o funcionamento do grupo de trabalho em uma produção do motor de Educação Física, propondo várias estratégias para melhorar os aspectos positivos do mesmo e minimizar os problemas mais comuns. Primeiro, ele explica a entrega do instrumento e avaliação e qualificação para ser usado com a intenção de treinamento. Em seguida, uma distribuição de papéis dentro do grupo é estabelecida para promover a responsabilidade individual. Subsequentemente, a produção do motor por meio de auto-avaliação, avaliação de pares entre grupos e heteroavaliação. Finalmente, ele vai recorrer a uma distribuição de notas para permitir que através de um coevaluacion intragrupo todos os alunos obter o grau que merecem.

Palavras-chave: Distribuir notas: trabalho de grupo; avaliação objectiva; ferramenta de avaliação

\section{Introducción}

Todo docente y alumno que haya realizado trabajos grupales sabe de los problemas que dichas propuestas suelen provocar (Velázquez, 2013): alumnos que se benefician del trabajo de otros o "polizones" (López-Pastor y Pérez-Pueyo, 2017), grupos que se perjudican por el bajo rendimiento de alguno de sus miembros, problemas de coordinación intragrupales y falta de identidad cuando se trata de buscar "culpables" ya que en ningún momento se ha establecido cual es el rol de cada miembro. 
A pesar de estos posibles inconvenientes, no son pocos quienes reconocen la potencialidad de los trabajos grupales para desarrollar habilidades de discusión, diálogo, respeto de las diferencias y otros muchos aprendizajes sociales y afectivos que este tipo de propuestas estimulan (Coll, 1984). No debemos olvidar que desde todas las asignaturas deberíamos contribuir al potencial desarrollo de todas las capacidades del individuo: intelectuales, físicas, afectivas y sociales (Pérez-Pueyo, 2005).

Es por ello que la solución no pasa por eliminar los trabajos grupales, sino por idear sistemas que eviten estos injustos abusos de unos estudiantes hacia otros.

\section{Contextualización}

La propuesta se llevó a cabo con los alumnos del IES Lancia (León) de toda la etapa de la ESO y de $1^{\circ}$ de Bachillerato en la asignatura de Educación Física. Tras realizar una unidad didáctica que culminaba con un trabajo grupal y una producción motriz se estableció un sistema de evaluación en el cual se aunaban: autoevaluación, coevaluación intergrupal y heteroevaluación.

En el proceso de calificación, se realizó un filtrado eliminando aquellas notas que obviamente no habían realizado una valoración justa de la producción motriz (eliminando notas con más de dos puntos de diferencia con respecto a la nota media). Esta fase es esencial para evitar "abusos" de los sistemas de evaluación democrática, al menos en las primeras etapas en las que algunos siguen queriendo engañar al sistema aún sabiendo que esa valoración que han realizado es absolutamente injusta.

Finalmente se calculaba la nota final realizando la media entre la nota del propio grupo, la de los otros grupos y la del profesor (33\% cada una). La nota final se multiplicaba por el número de personas del grupo y se obtenían los puntos a repartir.

\section{Diseño y desarrollo}

Para que el sistema de evaluación fuera justo y transparente se entregó el instrumento de evaluación (escala de valoración) en la primera sesión de la unidad didáctica. Los alumnos fueron informados de que aunque el trabajo era grupal y se valoraría como tal, todos y cada uno de ellos obtendrían calificaciones distintas en función de su trabajo diario y contribución a la producción motriz. También se establecieron papeles a desempeñar dentro de cada grupo (líder, comunicador, música, comportamiento, 
secretario), otorgando así responsabilidades individuales que eviten las excusas colectivas.

De la misma manera se les entregó un diario de seguimiento en el que día a día el/los secretario/s del grupo valoraban el trabajo de todos los miembros (coevaluación intragrupal). Precisamente este instrumento nos permitirá realizar un reparto de notas más fundamentado en situaciones objetivas y menos en el status social de los distintos componentes.

\section{Evaluación y conclusiones}

Dentro de los muchos sistemas de reparto de notas existentes (Morales, 2008; PérezPueyo, Hortigüela y Hernando 2013), en este caso se optó por multiplicar la nota final por el número de miembros del grupo (Nota 7,5 x 5 miembros = 37,5 puntos) en primer lugar para luego proceder al reparto de esos puntos. Dos de los criterios que se debían seguir eran: (1) que todos tuvieran calificaciones distintas (diferencia mínima de 0,1 puntos), y (2) que todos los miembros estuvieran de acuerdo en la calificación recibida en base a lo registrado en el diario de seguimiento y las aportaciones diarias.

En caso de no haber acuerdo, el líder de cada grupo podría decidir la calificación final.

Se generaron múltiples situaciones tanto positivas como negativas:

- Entre las positivas podemos destacar: la argumentación de cada alumno para justificar que se valorase su trabajo, la coevaluación de la aportación de los miembros del grupo, la similitud de las calificaciones obtenidas por las tres vías y que ningún grupo percibiera su calificación como injusta.

- Entre las negativas resulta llamativo que en varios grupos se asignaron las notas por estatus dentro del grupo independientemente de los instrumentos de evaluación aportados. Además algunos alumnos (los que menos habían aportado) se quejaron de las diferencias en las notas finales. Precisamente se aprovecharon estas situaciones para dialogar con los alumnos y demostrar que quizás no estamos acostumbrados a procesos objetivos de evaluación y calificación.

La experiencia resultó muy favorable ya que no sólo permitió que comprendieran en qué consiste un verdadero trabajo grupal sino que provocó que se 
creará una dinámica de trabajo mucho más justa en la que todos los obtenían lo que merecían, no con un fin únicamente sumativo sino buscando restablecer el sistema social del aula. Por otro lado, siempre en busca de un proceso de evaluación formativo resaltar que siguiendo este método los resultados son mejores ya que ambos instrumentos de evaluación (escala de valoración de la producción motriz y diario de seguimiento) les permiten entender que se espera de su producción final y de su participación individual dentro del grupo.

\section{Referencias}

Coll, C. (1984). Estructura grupal, interacción entre alumnos y aprendizaje escolar. Infancia y aprendizaje, 7(27-28), 119-138.

López-Pastor, V.M., \& Pérez-Pueyo, A. (Coord) (2017). Buenas prácticas docentes. Evaluación formativa y compartida en educación: experiencias en todas las etapas educativas. Servicio de publicaciones. Universidad de León.

Morales, P. (2008). Estrategias para evaluar y calificar el producto del equipo: cómo diferenciar las calificaciones individuales. En Prieto, L. (Coord.). La enseñanza centrada en el aprendizaje: estrategias útiles para el profesorado (pp.151-169). Barcelona: Octaedro.

Pérez- Pueyo, A. (2005). Estudio del planteamiento actitudinal del área de educación física de la Educación Secundaria Obligatoria en la LOGSE: Una propuesta didáctica centrada en una metodología basada en actitudes. Tesis doctoral. Servicio de Publicaciones. Universidad de León.

Pérez-Pueyo, A., Hortigüela, D. \& Hernando, A. (2013). La coevaluación intragrupal y el reparto de notas bajo un proceso de evaluación formativa. En Membiela, P., Casado, N. \& Cebreiros M.I. (2014). Experiencias e innovación docente en el contexto actual de la docencia universitaria (pp. 285-289). Orense. Educación Editora.

Velázquez, C. (2013). Análisis de la implementación del aprendizaje cooperativo durante la escolarización obligatoria en el área de Educación Física. Tesis doctoral sin publicar. Universidad de Valladolid. 